\title{
Effects of the Hydroalcoholic Extract of Zingiber officinale on Arginase I Activity and Expression in the Retina of Streptozotocin-Induced
} Diabetic Rats

\author{
Nasrin Lamuchi-Deli, ${ }^{1}$ Mohammad Aberomand, ${ }^{1}$ Hossein Babaahmadi-Rezaei, ${ }^{1}$ and Ghorban \\ Mohammadzadeh ${ }^{2, *}$ \\ ${ }^{1}$ Department of Clinical Biochemistry, Faculty of Medicine, Ahvaz Jundishapur University of Medical Sciences, Ahvaz, IR Iran \\ ${ }^{2}$ Hyperlipidemia Research Center, Department of Clinical Biochemistry, Faculty of Medicine, Ahvaz Jundishapur University of Medical Sciences, Ahvaz, IR Iran \\ "Corresponding author: Ghorban Mohammadzadeh, Hyperlipidemia Research Center, Department of Clinical Biochemistry, Faculty of Medicine, Ahvaz Jundishapur \\ University of Medical Sciences, Ahvaz, IR Iran. Tel: +98-09113436812, Fax: +98-6133332036, E-mail: mohammadzadeh@ajums.ac.ir
}

Received 2016 September 11; Revised 2017 January 23; Accepted 2017 February 01.

\begin{abstract}
Background: Emerging evidence suggests that an increased arginase activity is involved in vascular dysfunction in experimental animals. Zingiber officinale Roscoe, commonly known as ginger, has been widely used in the traditional medicine for treatment of diabetes.

Objectives: This study aimed at investigating the effects of the hydroalcoholic extract of Z. officinale on arginase I activity and expression in the retina of streptozotocin (STZ)-induced diabetic rats.

Methods: In this experimental study, 16 male Wistar rats weighing $200-250$ g were assessed. Diabetes was induced via a single intraperitoneal injection of STZ ( $60 \mathrm{mg} / \mathrm{kg}$ body weight). The rats were randomly allocated into four experimental groups. Untreated healthy and diabetic controls received $1.5 \mathrm{~mL} / \mathrm{kg}$ distilled water. Treated diabetic rats received 200, and $400 \mathrm{mg} / \mathrm{kg}$ of the Z. officinale extract dissolved in distilled water $(1.5 \mathrm{~mL} / \mathrm{kg})$. Body weight, blood glucose and insulin concentration were measured by standard methods. The arginase I activity and expression were determined by spectrophotometric and western blot analysis, respectively. Results: Our results showed that blood glucose concentration was significantly decreased in diabetic rats treated with the extract compared to untreated diabetic controls $(\mathrm{P}<0.01)$. Treatment with $400 \mathrm{mg} / \mathrm{kg}$ of the extract reduced arginase I activity and expression $(\mathrm{P}<0.05)$. A significant elevation in body weight was observed in diabetic rats treated with the extract. Serum insulin was significantly increased in diabetic rats treated with $400 \mathrm{mg} / \mathrm{kg}$ of the extract compared to diabetic controls $(\mathrm{P}<0.05)$.

Conclusions: Our results suggest that the $Z$. officinale hydroalcoholic extract may potentially be a promising therapeutic option for treating diabetes-induced vascular disorders, possibly through reducing arginase I activity and expression in the retina.
\end{abstract}

Keywords: STZ-Induced Diabetes, Arginase, Z. officinale, Hydroalcoholic Extract

\section{Background}

Emerging evidence suggests that enhanced arginase activity and expression is involved in the conditions characterized by vascular endothelial dysfunction including hypertension, atherosclerosis, diabetes, and aging both in experimental animal models $(1,2)$ and patients with diabetes (2-4). Nitric oxide synthase (NOS), in the vascular endothelial cells, consumes L-arginine (L-Arg) to produce NO, which regulates blood flow and decreases inflammation (5, 6). Decreased availability of L-Arg to NOS and subsequently reduction of NO production have been contributed to the vascular dysfunction associated with diabetes. Deficiency in the L-Arg availability for NOS can occur by over expression or increased activity of arginase, an enzyme that competes with NOS for the use of common substrate, L-Arg (7). Arginase(L-Arginine urea hydrolase, EC
3.5.3.1), which catalyzes the hydrolysis of L-arginine to urea and L-ornithine, exists in two isoforms, the hepatic type, arginase I, and the extra-hepatic type, arginase II, which are found within cytosol and mitochondria, respectively (7-9) and are found in endothelial cell populations $(4,7)$. It has been reported arginase I activity is enhanced in diabetic retinopathy, asthma and sickle cell disease, while arginase II has been implicated specifically in retinopathy of premature atherosclerosis and diabetic renal injury(10). Studies have shown that vascular dysfunction in diabetes is directly correlated with oxidative stress and inflammation (11, 12), both of which have been correlated with upregulation of arginase activity and expression $(13,14)$. In fact, inflammatory molecules, which are increased in hyperglycaemia including cytokines, oxidative radicals and glucose, activate the arginase pathway, whereas, simultaneously, they can reduce the eNOS pathway $(7,15,16)$. There- 
fore, L-Arg pool insufficiency can cause the NOS homodimer to become uncoupled, leading to produce superoxide. Superoxide and any available NO can interact to form a strong biological oxidant, peroxy-nitrite anion (ONOO-), further inducing excessive oxidative stress and less NO production $(14,17)$. Peroxynitrite can mediate cellular damage via direct oxidative interactions with lipids, DNA and proteins or via indirect (17). On the other hand, an excessive activity of the arginase pathway participates in promoting abnormal vascular growth and stiffness through increasing production of polyamines and proline (18). This offers that arginase can be a therapeutic target for diabetic retinopathy.

Zingiber Officinal Roscoe (Z. officinale), a widely-utilized spice worldwide, has been commonly considered as a traditional herbal medicine, exhibiting antioxidant, antiinflammatory, and antidiabetic properties (19-21). It also possesses protective effects on diabetic complications (19). In addition, some in vivo and in vitro studies have reported that Z. officinale can delay the development of diabetic cataract (22-24). According to the aforementioned, we hypothesized that $Z$. officinale might diminish arginase activity. This study aimed at investigating the effect of the Z. officinale methanolic extract on the arginase activity and its protein level in vivo using the streptozotocin (STZ)induced diabetic rat model.

\section{Objectives}

The current study was designed to determine the effects of the hydroalcoholic extract of $Z$. officinale on the arginase I activity and expression in the retina of STZinduced diabetic rats.

\section{Methods}

\subsection{Animals}

A total 16 male Wistar rats with an average weight of 200 - $250 \mathrm{~g}$ ( 8 - 10 weeks of age) were studied. The rats were purchased from the research center and experimental animal house of Ahvaz Jundishapur University of Medical Sciences (Ahvaz, Iran). The study was approved in accordance with the ethical committee of animal breeding and research of Jundishapur University of Medical Sciences (approval number: MPRC-9406, approval date: 2015/7/1). Upon arrival, the animals were housed in a well-ventilated room with a relative temperature of $22 \pm 2^{\circ} \mathrm{C}$ and photoperiod (12-hour light/dark cycle). Animals were given free access to standard food pellet and tap water during the experiment.

\subsection{Experimental Design}

After one-week adaptation, following an overnight fasting, type 1 diabetes mellitus was induced by a single intraperitoneal injection of STZ (Sigma-Aldrich, USA, 60 $\mathrm{mg} / \mathrm{kg}$, freshly dissolved in $0.1 \mathrm{M}$ cold citrate buffer, $\mathrm{pH}$ 4.5) (25). The healthy control group was injected with the same volume of the sodium citrate buffer solution alone. Three days after STZ administration, the rats, which have nicked tail-vein blood glucose concentration more than $350 \mathrm{mg} / \mathrm{dL}$ were considered diabetic. Oral gavage of the Z. officinale extract was initiated two weeks after the STZ injection and continued for a period of 8 weeks. The rats were randomly categorized into four experimental groups of four rats in each group as follows:

Group 1: The untreated healthy control group was gavaged with $1.5 \mathrm{~mL} / \mathrm{kg}$ distilled water once daily for 8 weeks. Group 2: The untreated diabetic control group gavaged with $1.5 \mathrm{~mL} / \mathrm{kg}$ distilled water once daily for 8 weeks. Group 3: Diabetic rats gavaged orally with the $Z$. officinale extract at a dose of $200 \mathrm{mg} / \mathrm{kg}$ body weight in distilled water (1.5 $\mathrm{mL} / \mathrm{kg}$ ) once daily for 8 weeks. Group 4: Diabetic rats gavaged orally with the Z. officinale extract at a dose of 400 $\mathrm{mg} / \mathrm{kg}$ body weight in distilled water $(1.5 \mathrm{~mL} / \mathrm{kg})$ once daily for 8 weeks.

\subsection{Sample Collection}

At the end of the eight-week treatment, the fasted rats in all groups were weighed and then sacrificed after anesthetizing. Fresh blood samples were directly obtained via cardiac puncture using not heparinized syringes. Subsequently the eye balls were immediately collected and washed in cold normal saline solution, then snap-frozen in liquid nitrogen and stored at $-80^{\circ} \mathrm{C}$ for later assay.

\subsection{Biochemical Analysis}

Sera were separated by centrifugation of whole blood at $3000 \mathrm{~g}$ for 15 minutes at $4^{\circ} \mathrm{C}$ and used for measurement of serum glucose and insulin concentration. Fasting blood glucose concentration was determined using the commercial available colorimetric kit (Pars Azmoon co, Tehran, Iran) with an automatic biochemical analyzer. Serum insulin concentration was determined using a commercial available rat insulin ELISA kit (Thermo Scientific Inc., Rockford, IL).

\subsection{Preparation of the Zingiber officinale Methanolic Extract}

Dried roots of Z. officinale (Z. officinale, family Zingiberaceae) were obtained from Gol Darou Company, Isfahan, Iran. The hydroalcoholic extract of $Z$. officinale was made through the maceration method. For extraction, dried $Z$. officinale roots (200 g) were powdered using an electric 
blender, soaked in $1400 \mathrm{~mL}$ of $70 \%$ methanol solution (v/v), and then kept at the room temperature for 72 hours. The infusion was then filtered through the Wattman filter no.4 and the solvent was evaporated using a rotary evaporator. To increase the shelf life and homogeneity, the extract was lyophilized completely by a continuous freeze-drying operation and the yield was $12.5 \%(\mathrm{w} / \mathrm{w})$, which was stored at $-20^{\circ} \mathrm{C}$ until use (26).

\subsection{Assay of Arginase Activity}

The assay was conducted by spectrophotometric measurement of urea production to confirm the efficacy of the $Z$. officinale effect on the arginase activity in the retina lysates. Briefly, first the whole eyes of rats were gently harvested and the retinas were peeled away. Then retina were sonicated in ice-cold lysis buffer containing $50 \mathrm{mM}$ Tris$\mathrm{HCl}$ (pH 7.5), 0.1 mM EDTA, 0.1 mM EGTA, 0.1\% TritonX-100 and freshly dissolved protease inhibitor. The samples were subsequently lysed by repeated freezing and thawing (3 cycles) and the lysates were then centrifuged at $16000 \mathrm{~g}$ at $4^{\circ} \mathrm{C}$ for $20 \mathrm{~min}$ to remove insoluble materials. Afterwards, $25 \mu \mathrm{L} \mathrm{MnCl}_{2}$ (10 mM in $50 \mathrm{Mm}$ Tris- $\mathrm{HCl}$ ) was added to 25 $\mu \mathrm{L}$ supernatant and heated at $56^{\circ} \mathrm{C}$ for $10 \mathrm{~min}$ to activate the enzyme. Next, to initiate enzyme activity, assay solution was added to $50 \mu \mathrm{L} \mathrm{L-Arg}$ (0.5 M in $50 \mathrm{mM}$ Tris-HCl, $\mathrm{pH}$ 9.7) and then incubated at $37^{\circ} \mathrm{C}$ for 1 hour. The arginase reaction was stopped by adding $400 \mu \mathrm{L}$ of an acid mixture $\left(\mathrm{H}_{2} \mathrm{SO}_{4}: \mathrm{H}_{3} \mathrm{PO}_{4}: \mathrm{H}_{2} \mathrm{O}\right.$ in a 1:3:7 ratio). For colorimetrical determination of urea, the mixture reaction was heated with $25 \mu \mathrm{L}$ of chromogenic $\alpha$-isonitrosopropiophenone (9\% in ethanol) at $100^{\circ} \mathrm{C}$ for 45 minutes and then placed in the dark at room temperature for 10 minutes. Finally, the absorbance was read by a spectrophotometer at a wavelength of $540 \mathrm{~nm}$. Arginase activity was expressed as picomoles of urea production per milligram of protein per hour. The blank solution contains all of the above-mentioned compounds except $\mathrm{MnCl} 2$ or L-Arg.

\subsection{Western Blot Analysis}

Western blot analysis was performed on the supernatant fraction of homogenized rat retinas. Total protein concentrations of the supernatant were determined by the Bradford method. Fifty micrograms (50 $\mu \mathrm{g}$ ) protein from each sample was loaded onto $10 \%$ odium dodecyl sulfate (SDS)-polyacrylamide gels for electrophoresis (SDS-PAGE). Protein blots were then electro- transferred to a methanolpreactivated polyvinylidene difluoride (PVDF) membrane in Tris-glycine buffer by BIO-RAD transfer system (USA). The membrane was blocked with bovine serum albumin (BSA) 5\% in Tris-buffered saline plus 0.1\% Tween-20 (TBST) for 1 hour with gently shakes. Subsequently, blots were probed with antibodies specific for Arg-I (1:200 dilution) (sc-20150; Santa Cruz Biotechnology), and then reprobed for $\beta$-actin (sc-130656; Santa Cruz Biotechnology) to ensure equal protein loading. Membrane bound antibody was incubated with secondary antibody, goat anti-rabbit IgG-HRP (sc-2030; Santa Cruz) diluted 1:10000 in 3\% BSA for 1 hour at the room temperature. After each step, membranes were washed thrice with TBST. Finally, the blots were visualized with the Chemi-Doc gel documentation system (BioRad, Hercules, CA) using an enhanced chemiluminescence (ECL) western blotting detection kit according to the manufacturer's protocol. Optical densities of bands were measured using Image J software and quantified as the ratio to $\beta$-actin. The mean value of samples for the healthy control rats in each blotting, expressed as the densitometry unit was adjusted to a value of 1.0. All experimental sample values were expressed relative to this adjusted mean value.

\subsection{Statistical Analysis}

All statistical analyses were performed with the SPSS software (SPSS Inc, Chicago, Illinois, USA). Results were reported as means \pm standard deviation. A value of $\mathrm{P}<0.05$ was regarded as statistically significant. One-way ANOVA followed by Tukey's post-hoc test was used for statistical comparisons of data with normal distribution (including blood glucose and body weight) and data with no normal distribution (including insulin, arginase activity, and expression) were analyzed by the Kruskal-Wallis test. Illustrations were done using the GraphPad Prism software (GraphPad Software Inc, San Diego, CA, USA).

\section{Results}

All rats in the four studied groups remained active throughout the experimental period. Before diabetes induction, there was no statistically significant difference in the body weight and fasting blood glucose concentration among four groups. The effects of the Z. officinale extract on body weight, fasting serum insulin and glucose, arginase activity, and arginase I protein level was ascertained by comparison of diabetic controls with healthy controls and treated diabetic with untreated diabetic controls.

\subsection{Effects of Zingiber officinale Extract on the Body Weight}

As shown in Table 1, at the end of the study, STZ-induced diabetes resulted in a significant reduction of body weight in the untreated diabetic controls compared to the healthy controls $(\mathrm{P}<0.01)$. However, body weight was significantly elevated in both treated diabetic groups compared to the 
untreated diabetic controls and this effect was more obvious in diabetic rats which received $400 \mathrm{mg} / \mathrm{kg}$ of the extract than those received $200 \mathrm{mg} / \mathrm{kg}$ of the extract $(\mathrm{P}<0.01$ and $\mathrm{P}<0.05$, respectively).

\subsection{Effects of the Zingiber officinale Extract on the Biochemical Parameters}

As expected and shown in Table 1, fasting serum insulin was significantly decreased in the STZ-induced diabetic rats compared to the healthy controls $(\mathrm{P}<0.01)$. Conversely, treatment with $400 \mathrm{mg} / \mathrm{kg}$ of the Z. officinale extract for eight weeks was significantly increased fasting serum insulin compared to the diabetic controls $(\mathrm{P}<$ 0.05). However, no statistically significant difference was observed in the fasting serum insulin concentration between diabetic treated with $200 \mathrm{mg} / \mathrm{kg}$ of the Z. officinale extract and diabetic controls $(\mathrm{P}=0.08)$.

As shown in Table 1, the untreated diabetic rats showed a markedly higher fasting blood glucose concentration than the healthy control group $(\mathrm{P}<0.01)$. While, after an eight-week treatment, blood glucose was significantly decreased in both $Z$. officinale treated diabetic groups compared to the untreated diabetic rats $(\mathrm{P}<0.01)$. In addition, consumption of $Z$. officinale at a daily dose of $400 \mathrm{mg} / \mathrm{kg}$ led to a greater effect in decreasing the fasting blood glucose concentration, which was not statistically different when compared to healthy controls $(\mathrm{P}=0.17)$. This indicates that $400 \mathrm{mg} / \mathrm{kg} \mathrm{Z}$. officinale treatment for 8 weeks normalized the fasting blood glucose concentration. Results are represented in Table 1.

\subsection{Effects of the Zingiber officinale Extract on the Activity and Protein Level of Arginase I}

As illustrated in Figure 1, at the end of the experimental period, a significant increase was observed in the arginase activity (109.6\%) in the untreated diabetic rats compared to the healthy controls $(\mathrm{P}<0.01)$. However, treatment with $400 \mathrm{mg} / \mathrm{kg}$ of the extract resulted in a remarkable decrease in arginase activity compared to the untreated diabetic controls $(\mathrm{P}<0.05)$. While, no statistically significant difference in the arginase activity was observed between diabetic rats treated with $200 \mathrm{mg} / \mathrm{kg}$ of the extract and untreated diabetic controls $(\mathrm{P}=0.21)$. Results are reported as percentage of control.

Similarly, the western blot analysis exhibited that the arginase I protein level was significantly increased (3.2 fold) in the retina of the STZ-induced diabetic rats compared to the healthy controls $(\mathrm{P}<0.01)$. Whereas, a significant reduction was observed in the arginase I protein level in diabetic rats treated with 200 and $400 \mathrm{mg} / \mathrm{kg}$ of Z. officinale extract as compared to the untreated diabetic

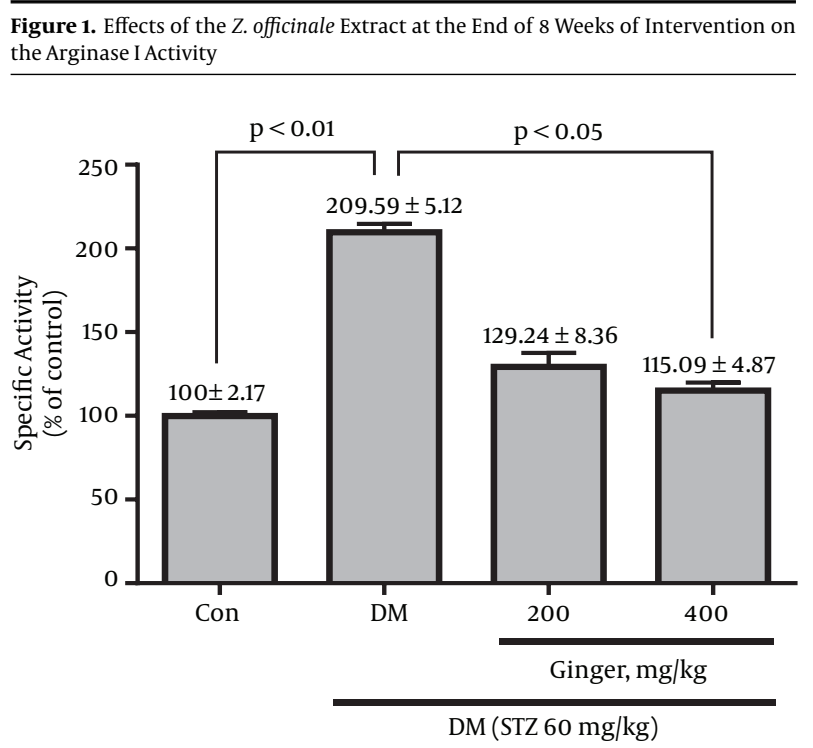

Arginase activity was measured as explained in the "methods" section. The percent age of the mean arginase I specific activity in each treated group was calculated relative to the control. Data are expressed as mean $\pm S D(n=4)$.

controls, more effect was observed in rats treated with 400 $\mathrm{mg} / \mathrm{kg}$ of the extract $(\mathrm{P}<0.01)$ (Figures $2 \mathrm{~A}$ and $2 \mathrm{~B}$ ).

\section{Discussion}

Despite major progress for hyperglycemia control through diet therapy, pharmacological agents, insulin and islet transplantation, management of long-term diabetic complications such as blindness still remains a major clinical challenge (23). Arginase, a key enzyme of urea cycle, mainly involved both in vascular and neuronal damage and the arginase/polyamine pathway plays an important role in the diabetic neurovascular damage in the retina (17).

Since some conventional medications marketed for diabetic complications have troublesome side-effects, interest in natural complementary and alternative therapies is increasing. In traditional medicine, plants have been considered as a rich source of therapeutic compounds for many indications. A number of these herbal medicines may be promising as therapies for diabetes and hyperglycemia, an important one is $Z$. officinale the blood glucose lowering effect of which has been observed and supported by recent in vivo and in vitro studies (19-24). Since no study regarding the effects of $Z$. officinale hydroalcoholic extract on the arginase expression and activity in diabetes has ever been performed, the current study aimed at examining the effects of Z. officinale hydroalcoholic extract, specifically in an animal model. 
Table 1. Effects of the Zingiber officinal Extract on the Body Weight, Insulin and Glucose Concentration in the Studied Groups ${ }^{\mathrm{a}}$

\begin{tabular}{lccc}
\hline Variables & Healthy Control & Diabetic Control & DM+ 200, mg/kg \\
\hline Body weight, $\mathbf{g}$ & $256.25 \pm 16.25$ & $171.00 \pm 15.29^{\mathrm{b}}$ & $200.75 \pm 14.63^{\mathrm{c}}$ \\
Serum insulin, $\mu \mathbf{U} / \mathbf{m L}$ & $23.77 \pm 0.38$ & $0.23 \pm 0.10^{\mathrm{b}}$ & $0.62 \pm 0.18$ \\
Serum glucose, $\mathbf{m g} / \mathbf{d L}$ & $155.25 \pm 10.14$ & $533.50 \pm 36.09^{\mathrm{b}}$ & $318.25 \pm 25.48^{\mathrm{d}}$ \\
\hline
\end{tabular}

Abbreviation: DM, diabetes mellitus.

${ }^{\mathrm{a}}$ Values are expressed as mean $\pm \mathrm{SD}(\mathrm{n}=4)$.

${ }^{\mathrm{b}} \mathrm{P}<0.01$ vs. healthy control.

${ }^{\mathrm{c}} \mathrm{P}<0.05$ vs. diabetic control.

${ }^{\mathrm{d}} \mathrm{P}<0.01$ vs. diabetic control.

Figure 2. Effects of the Z. officinal Extract at the End of 8 Weeks of Intervention on the Arginase I Expression

\section{A}

Arginase I

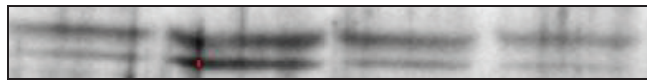

$\beta$ - Actin

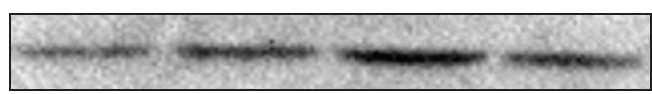

B

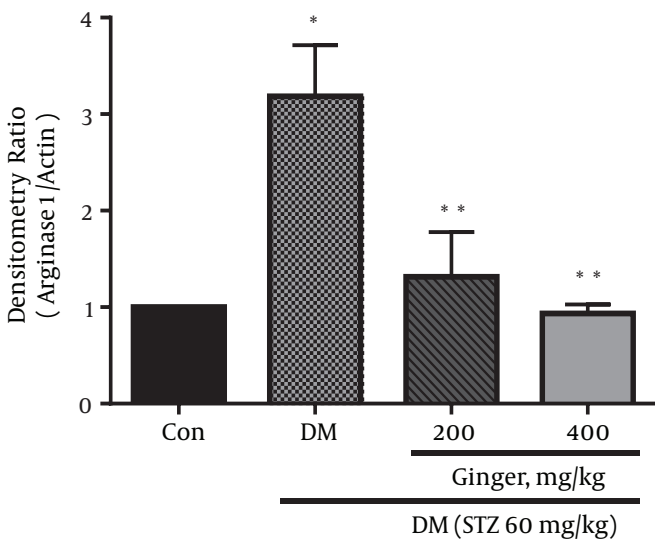

A, Representative immune-blots demonstrating specific bands for arginase I; B, Graphical presentation of data obtained from four independent experiments from the western blot analysis. The mean value of arginase I is expressed as the ratio of arginase I to $\beta$-actin in each column $(n=4)$. $\beta$-actin was applied as an internal control. Error bars show S.D. ${ }^{*} \mathrm{P}<0.05$ vs. healthy control; and ${ }^{* *} \mathrm{P}<0.05$ vs. diabetic controls.

Our results indicated that body weight significantly decreased in the untreated diabetic rats compared to the healthy controls. In a study conducted by Kusari et al. loss of body weight in the STZ-induced diabetic rats had been attributed either to the enhancement of urine output, which leads to dehydration and loss of valuable flu- ids, or to muscle breakdown resulting from hyperglycemia (27). Our results are also in agreement with the results of Eleazu et al. who reported the cause of weight loss in the STZ-induced-diabetic rats resulted from the loss and degradation of structural proteins (28). We found that the Z. officinale extract protects the STZ-induced diabetic rats against weight loss and this effect may be due to its potential hypoglycemic properties. Moreover, this result is in agreement with the findings reported by Faried et al. and Thomson et al. $(23,29)$.

In the current study, following 10 weeks of diabetes induction, serum insulin significantly decreased in the untreated diabetic controls compared to healthy controls. In line with these findings, Zafar et al. observed that the diabetogenic effect of STZ is a direct result of the irreversible damages of $\beta$ cells, which leads to degranulation and decrease of insulin secretion (30). In addition, our results showed that serum insulin in diabetic rats treated with $400 \mathrm{mg} / \mathrm{kg}$ of the Z. officinale extract significantly increased relative to the untreated diabetic group, results consistent with those reported by Iranloye et al., and Akhani et al. (31, 32 ); according to their results, they speculated that the $Z$. officinale extract may be attributed to increased insulin secretion from $\beta$ cells or release of granule-bounded insulin (31). Heimes et al. suggested that the effects of Z. officinale in the retrieval of insulin may be due to its reaction with the 5-Hydroxy-tripetamin (5-HT3) receptor (33). Several studies have confirmed that serotonin receptors may be involved in the hypoglycemic effects of the Z. officinale extract. Serotonin receptors suppress the release of insulin and the $Z$. officinale extract can antagonize this inhibitory effect (34).

We found that, accompanied by a considerable decrease in serum insulin, a significant increase in the fasting blood glucose concentration was observed in the untreated diabetic rats compared to healthy control, a result consistent with those reported elsewhere $(21,30,31)$. Increased blood glucose concentration can be due to dysfunction of insulin secretion following STZ-induced dia- 
betes (35). On the other hand, we found that reduction of fasting blood glucose in diabetic rats treated with the Z. officinale extract was dose-dependent, a result in accordance with those reported by several studies $(21,22,29)$. The antihyperglycemic property of $Z$. officinal is possibly due to its phenols, polyphenol substances and flavonoids (19). Previous studies suggested that the effect of the Z. officinale extract on the reduction of blood glucose may be mediated by decreasing the absorption of glucose through inhibiting the activity of intestinal $\alpha$-amylase and $\alpha$-glucosidase (36), and its antagonistic activity against serotonin receptors and blocking of them (37).

Reduced blood flow into the retina is one of the most adverse consequences of the vascular dysfunction in diabetic retinopathy (1-3). Several signaling pathways are involved in the vascular dysfunction in diabetes, including, protein kinase $\mathrm{C}$ ( $\mathrm{PKC}$ ), endothelin, angiotensin II, and NO. Also, according to the recent studies, increased activity of arginase, induced by diabetes, plays a primary role in an impaired endothelium-dependent vasodilation response (4). Combinations of these mechanisms contribute to the development of diabetic complications (23). Previous studies conducted about the mediators, which impair retinal blood flow induced by diabetes, have demonstrated an important role for PKC and angiotensin II in changing retinal vasodilation responses. Activation of both PKC and angiotensin II pathways not only increases ROSs, but also leads to increased activity of arginase in vascular tissue. In addition, it has been showed that PKC inhibits the endothelium-dependent vasodilation of the retinal arteries. The protein kinase $\mathrm{C}$ can also activate the RhoA/Rho kinase signaling pathway in endothelial cells; RhoA/Rho kinase is one of the upstream regulators of arginase. Therefore, it seems that arginase is the last connector of various mediators of vascular injuries in the retina of diabetic individuals and this emphasizes the potential importance of arginase as a therapeutic target for treatment of diabetic retinopathy (4). It has been proved that oxidative stress and inflammatory reactions increase in the diabetic conditions (16). Moreover, various inflammatory factors and oxidative radicals increase arginase activity (15). Our results revealed that arginase activity and expression significantly increased in the retina of the untreated diabetic controls compared to the healthy control group, findings similar those reported by Elms et al. (4). On the other hand, our results indicated that treatment with $400 \mathrm{mg} / \mathrm{kg}$ of the Z. officinale hydroalcoholic extract resulted in a significant increase in arginase activity and expression, results which can be explained by the antiinflammatory, antioxidant and antihyperglycemic properties of the Z. officinal extract; that inhibit arginase activators (i.e. inflammatory factors, oxidative radicals and glucose). According to previous studies, Z. officinale has a compound named 6shogaol, which possesses the most antioxidant and antiinflammatory effects (19). On the other hand, many in vivo and in vitro studies have documented the beneficial effects of Z. officinale extract on the improvement of diabetic retinopathy (22-24).

To the best of our knowledge, our study is the first to address the effect of the Z. officinale hydroalcoholic extract on the arginase I activity and expression in the retina of STZinduced diabetic rats. This interventional study was conducted in a sample of the STZ-induced diabetic rats; thus, clinical outcomes may be expected in further studies. However, the small sample size is a limitation in the current study and the results should be interpreted with caution. Further studies should consider replicating these results with larger sample sizes.

Conclusion: According to recent studies, the role of the arginase I has been reported in the diabetic-induced retinal vascular dysfunction $(1,10)$. Our results indicated that the $Z$. officinale hydroalcoholic extract may be a promising therapeutic potential for treating vascular disorders associated with diabetes possibly through its reducing effect on the arginase I activity and expression.

\section{Acknowledgments}

This paper was issued from the thesis of Nasrin Lamuchi-Deli and financial support was provided by the medicinal plant research center, Ahvaz Jundishapur University of Medical Sciences (grant no. MPRC-9406).

\section{References}

1. Patel C, Rojas M, Narayanan SP, Zhang W, Xu Z, Lemtalsi T, et al. Arginase as a mediator of diabetic retinopathy. Front Immunol. 2013;4:173. doi: 10.3389/fimmu.2013.00173. [PubMed: 23840196].

2. Yao L, Chandra S, Toque HA, Bhatta A, Rojas M, Caldwell RB, et al. Prevention of diabetes-induced arginase activation and vascular dysfunction by Rho kinase (ROCK) knockout. Cardiovasc Res. 2013;97(3):509-19. doi:10.1093/cvr/cvs371. [PubMed: 23250919].

3. Shemyakin A, Kovamees O, Rafnsson A, Bohm F, Svenarud P, Settergren $\mathrm{M}$, et al. Arginase inhibition improves endothelial function in patients with coronary artery disease and type 2 diabetes mellitus. Circulation. 2012;126(25):2943-50. doi: 10.1161/CIRCULATIONAHA.112.140335. [PubMed: 23183942].

4. Kovamees O, Shemyakin A, Pernow J. Effect of arginase inhibition on ischemia-reperfusion injury in patients with coronary artery disease with and without diabetes mellitus. PLoS One. 2014;9(7):ee103260. doi: 10.1371/journal.pone.0103260. [PubMed: 25072937].

5. Kuhlencordt PJ, Gyurko R, Han F, Scherrer-Crosbie M, Aretz TH, Hajjar R, et al. Accelerated atherosclerosis, aortic aneurysm formation, and ischemic heart disease in apolipoprotein E/endothelial nitric oxide synthase double-knockout mice. Circulation. 2001;104(4):448-54. [PubMed: 11468208]. 
6. Lefer DJ, Jones SP, Girod WG, Baines A, Grisham MB, Cockrell AS, et al. Leukocyte-endothelial cell interactions in nitric oxide synthasedeficient mice. Am J Physiol. 1999;276(6 Pt 2):H1943-50. [PubMed: 10362674].

7. Romero MJ, Iddings JA, Platt DH, Ali MI, Cederbaum SD, Stepp DW, et al. Diabetes-induced vascular dysfunction involves arginase I. Am J Physiol Heart Circ Physiol. 2012;302(1):H159-66. doi: 10.1152/ajpheart.00774.2011. [PubMed: 22058149].

8. Morris SJ. Regulation of enzymes of the urea cycle and arginine metabolism. Annu Rev Nutr. 2002;22:87-105. doi: 10.1146/annurev.nutr.22.110801.140547. [PubMed: 12055339].

9. Miyanaka K, Gotoh T, Nagasaki A, Takeya M, Ozaki M, Iwase K, et al. Immunohistochemical localization of arginase II and other enzymes of arginine metabolism in rat kidney and liver. Histochem J. 1998;30(10):741-51. [PubMed: 9874001].

10. Elms SC, Toque HA, Rojas M, Xu Z, Caldwell RW, Caldwell RB. The role of arginase I in diabetes-induced retinal vascular dysfunction in mouse and rat models of diabetes. Diabetologia. 2013;56(3):654-62. doi: 10.1007/s00125-012-2789-5. [PubMed: 23232640].

11. Cohen RA, Tong X. Vascular oxidative stress: the common link in hypertensive and diabetic vascular disease. J Cardiovasc Pharmacol. 2010;55(4):308-16. [PubMed: 20422735].

12. Tabit CE, Chung WB, Hamburg NM, Vita JA. Endothelial dysfunction in diabetes mellitus: molecular mechanisms and clinical implications. Rev Endocr Metab Disord. 2010;11(1):61-74. doi: 10.1007/s11154-010-91344. [PubMed: 20186491].

13. Bivalacqua TJ, Hellstrom WJ, Kadowitz PJ, Champion HC. Increased expression of arginase II in human diabetic corpus cavernosum in diabetic-associated erectile dysfunction. Biochem Biophys Res Commun. 2001;283(4):923-7. doi: 10.1006/bbrc.2001.4874. [PubMed: 11350073].

14. Jiang M, Jia L, Jiang W, Hu X, Zhou H, Gao X, et al. Protein disregulation in red blood cell membranes of type 2 diabetic patients. Biochem Biophys Res Commun. 2003;309(1):196-200. [PubMed: 12943682].

15. Pernow J, Jung C. Arginase as a potential target in the treatment of cardiovascular disease: reversal of arginine steal?. Cardiovasc Res. 2013;98(3):334-43. doi: 10.1093/cvr/cvt036. [PubMed: 23417041].

16. Bachetti T, Comini L, Francolini G, Bastianon D, Valetti B, Cadei $\mathrm{M}$, et al. Arginase pathway in human endothelial cells in pathophysiological conditions. J Mol Cell Cardiol. 2004;37(2):515-23. doi: 10.1016/j.yjmcc.2004.05.004. [PubMed: 15276021].

17. Narayanan SP, Rojas M, Suwanpradid J, Toque HA, Caldwell RW, Caldwell RB. Arginase in retinopathy. Prog Retin Eye Res. 2013;36:260-80. doi: 10.1016/j.preteyeres.2013.06.002. [PubMed: 23830845].

18. Caldwell RB, Zhang W, Romero MJ, Caldwell RW. Vascular dysfunction in retinopathy-an emerging role for arginase. Brain Res Bull. 2010;81(23):303-9. doi: 10.1016/j.brainresbull.2009.08.025. [PubMed: 19737603].

19. Li Y, Tran VH, Duke CC, Roufogalis BD. Preventive and protective properties of Zingiber officinale (ginger) in diabetes mellitus, diabetic complications, and associated lipid and other metabolic disorders: a brief review. Evid Based Complement Alternat Med. 2012;2012:1-10.

20. Rahmani AH, Shabrmi FM, Aly SM. Active ingredients of ginger as potential candidates in the prevention and treatment of diseases via modulation of biological activities. Int J Physiol Pathophysiol Pharmacol. 2014;6(2):125-36. [PubMed: 25057339].

21. Abdulrazaq NB, Cho MM, Win NN, Zaman R, Rahman MT. Beneficial effects of ginger (Zingiber officinale) on carbohydrate metabolism in streptozotocin-induced diabetic rats. Br J Nutr. 2012;108(7):1194-201. doi: 10.1017/S0007114511006635. [PubMed: 22152092].

22. Saraswat M, Suryanarayana P, Reddy PY, Patil MA, Balakrishna N,
Reddy GB. Antiglycating potential of Zingiber officinalis and delay of diabetic cataract in rats. Mol Vis. 2010;16:1525-37. [PubMed: 20806076].

23. Faried MA, Mansour FK, Zolfakar AS, El-Kholy WB. Protective Role of Ginger on the Induced Diabetic Retinopathy in Adult Albino Rats. $J$ Am Sci. 2014;10(10):134-52.

24. Kato A, Higuchi Y, Goto H, Kizu H, Okamoto T, Asano N, et al. Inhibitory effects of Zingiber officinale Roscoe derived components on aldose reductase activity in vitro and in vivo. J Agric Food Chem. 2006;54(18):6640-4. doi: 10.1021/jf061599a. [PubMed:16939321].

25. Tzeng TF, Liou SS, Chang CJ, Liu IM. Zerumbone, a tropical ginger sesquiterpene, ameliorates streptozotocin-induced diabetic nephropathy in rats by reducing the hyperglycemia-induced inflammatory response. Nutr Metab (Lond). 2013;10(1):64. doi: 10.1186/1743-7075-10-64. [PubMed: 24499158].

26. Bordbar H, Esmaeilpour T, Dehghani F, Panjehshahin MR. Stereological study of the effect of ginger's alcoholic extract on the testis in busulfan-induced infertility in rats. Iran J Reprod Med. 2013;11(6):46772. [PubMed: 24639780].

27. Kusari J, Zhou S, Padillo E, Clarke KG, Gil DW. Effect of memantine on neuroretinal function and retinal vascular changes of streptozotocin-induced diabetic rats. Invest Ophthalmol Vis Sci. 2007;48(11):5152-9. doi: 10.1167/iovs.07-0427. [PubMed: 17962468].

28. Eleazu CO, Iroaganachi M, Okafor PN, Ijeh I, Eleazu KC. Ameliorative Potentials of Ginger (Z. officinale Roscoe) on Relative Organ Weights in Streptozotocin induced Diabetic Rats. Int J Biomed Sci. 2013;9(2):8290. [PubMed: 23847458].

29. Al-Amin ZM, Thomson M, Al-Qattan KK, Peltonen-Shalaby R, Ali M. Anti-diabetic and hypolipidaemic properties of ginger (Zingiber officinale) in streptozotocin-induced diabetic rats. Br J Nutr. 2006;96(4):660-6. [PubMed: 17010224].

30. Zafar M, Naeem-ul-Hassan Naqvi S. Effects of STZ-Induced Diabetes on the Relative Weights of Kidney, Liver and Pancreas in Albino Rats: A Comparative Study. Int J Morphol. 2010;28(1) doi: 10.4067/s071795022010000100019.

31. Iranloye BO, Arikawe AP, Rotimi G, Sogbade AO. Anti-diabetic and anti-oxidant effects of Zingiber officinale on alloxan-induced and insulin-resistant diabetic male rats. Niger J Physiol Sci. 2011;26(1):8996. [PubMed: 22314994].

32. Akhani SP, Vishwakarma SL, Goyal RK. Anti-diabetic activity of Zingiber officinale in streptozotocin-induced type I diabetic rats.JPharm Pharmacol. 2004;56(1):101-5. doi: 10.1211/0022357022403. [PubMed: 14980006].

33. Heimes K, Feistel B, Verspohl EJ. Impact of the 5-HT3 receptor channel system for insulin secretion and interaction of ginger extracts. Eur J Pharmacol. 2009;624(1-3):58-65. doi: 10.1016/j.ejphar.2009.09.049. [PubMed: 19818348].

34. Salim KS. Hypoglycemic Property of Z. officinale and Green Tea and their Possible Mechanisms in Diabetes Mellitus. Open Conference Proc J. 2014;5:13-9.

35. Take G, Karabay G, Erdogan D, Duyar I. The ultrastructural alterations in rat corneas with experimentally-induced diabetes mellitus. Saudi Med J. 2006;27(11):1650-5. [PubMed: 17106535].

36. Shanmugam KR, Mallikarjuna K, Kesireddy N, Sathyavelu Reddy K. Neuroprotective effect of ginger on anti-oxidant enzymes in streptozotocin-induced diabetic rats. Food Chem Toxicol. 2011;49(4):893-7. doi: 10.1016/j.fct.2010.12.013. [PubMed: 21184796].

37. Goyal RK, Kadnur SV. Beneficial effects of Zingiber officinale on goldthioglucose induced obesity. Fitoterapia. 2006;77(3):160-3. doi: 10.1016/j.fitote.2006.01.005. [PubMed: 16513292]. 